\title{
Channeling of Aberration-corrected STEM Probes at the "Sub-atomic" Scale
}

\author{
Michael L. Odlyzko ${ }^{1}$ and K. Andre Mkhoyan ${ }^{1}$ \\ ${ }^{1}$ Chemical Engineering and Materials Science, University of Minnesota, Minneapolis, MN 55455
}

The phenomenon of fast electron channeling in atomic crystals has long been appreciated as an important factor in TEM characterization, being particularly critical for consideration in scanning transmission electron microscopy (STEM). As such, electron channeling effects have been examined to understand the thickness-dependence of annular-dark-field (ADF) STEM image contrast [1], the emergence of atomic-scale core-loss electron-energy-loss spectroscopy (EELS) spectrum image contrast [2], the orientation-dependence [3] and spatial localization [4] of X-ray energy dispersive spectroscopy (XEDS) signals, and the detectability of dopant atoms [5]; STEM imaging has, in turn, recently been used to experimentally measure electron channeling behavior [6].

The scattering of on-column intensity is the primary contributor to ADF and spectrum image signals in STEM, thus electron channeling studies of crystals have focused on the on-column intensity oscillations of converged probes centered on (or very slightly off of) atomic columns. However, to better understand the evolution of channeling behavior of fine aberration-corrected STEM probes when scanned over a crystal, we have employed multislice [7,8] simulations of probe propagation wherein a two-dimensional intensity map is saved at every z-slice for each incident probe position. The TEMSIM multislice package [9] was used to simulate aberration-corrected STEM probes (accelerating voltages 100-300 kV, probe convergence semi-angles 20-25 mrad) in various crystals, averaging over many frozen phonon configurations at room temperature. Simulation inputs were generated both by using the default parameterization tables of TEMSIM and by parameterizing projected atomic potentials calculated via the Quantum Espresso package [10].

Simulated data for $<100>$-oriented $\mathrm{SrTiO}_{3}$ (Figure 1) and $<2110>$-oriented wurtzite AlN (Figure 2) both exemplify the "sub-atomic" lateral complexity of channeling behavior, especially of off-column probes. Implications of such channeling behavior for high resolution ADF-STEM imaging, EELS and EDS spectrum imaging, and EELS fine structure features will also be discussed [11].

\section{References}

[1] P. Voyles et al., Ultramicroscopy 96 (2003), p. 251.

[2] L.J. Allen et al., Phys. Rev. Lett. 91 (2003), p. 105503.

[3] J.C.H. Spence and J. Tafte, J. Microsc. 130 (1983), p. 147.

[4] M.-W. Chu et al., Phys. Rev. Lett. 104 (2010), p. 196101.

[5] A. Mittal and K.A. Mkhoyan, Ultramicroscopy 111 (2011), p.1101.

[6] L. Fitting Kourkoutis et al., Phys. Rev. B 84 (2011), p. 075485.

[7] M.A. O'Keefe et al., Nature 274 (1978), p. 322.

[8] E.J. Kirkland et al., Ultramicroscopy 23 (1987), p. 77.

[9] E.J. Kirkland, Advanced Computing in Electron Microscopy, (Springer, New York, 2010).

[10] P. Giannozzi et al., J. Phys. Cond. Matter 29 (2009), p. 395502.

[11] This research was supported by NSF DMR-1006706. Simulations were performed using Minnesota Supercomputing Institute resources. Drs. M. Cococcioni and B. Himmetoglu are thanked for density functional theory calculations of bonding charge density. 


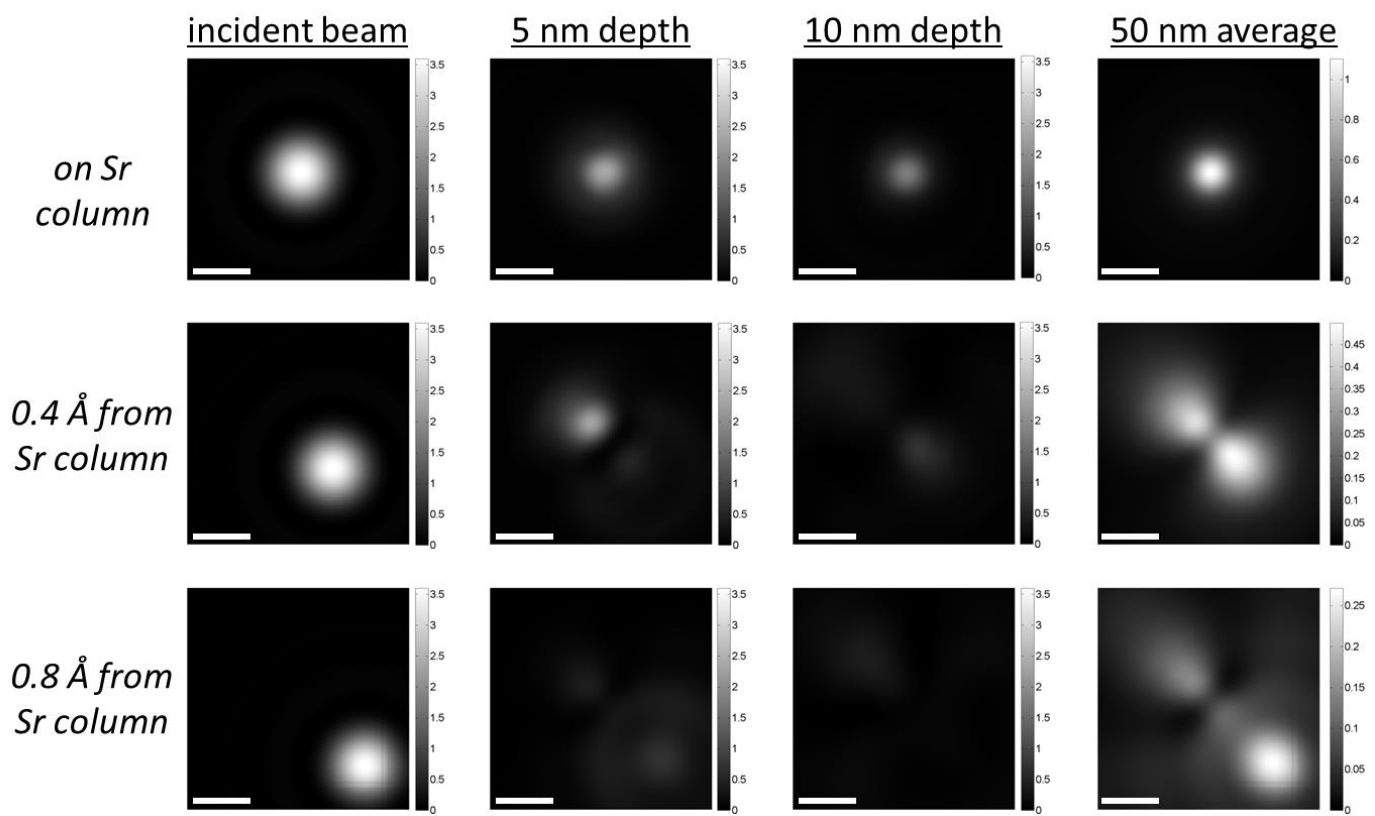

Figure 1. Intensity of a $300 \mathrm{kV}$ probe with convergence semi-angle $21.4 \mathrm{mrad}$ in $<100>$-oriented $\mathrm{SrTiO}_{3}$ at three different depths, as well as averaged over $50 \mathrm{~nm}$ depth (scale bar length $0.5 \AA$, intensity in units of normalized flux $\AA^{2}$ ). The on-column probe remains focused on the column throughout, but both of the above off-column probes oscillate in intensity on either side of the column in a "dumbbell" shape along the line connecting the incident position and the column; note also the local minimum in depth-averaged intensity at the column position for the off-column probes.

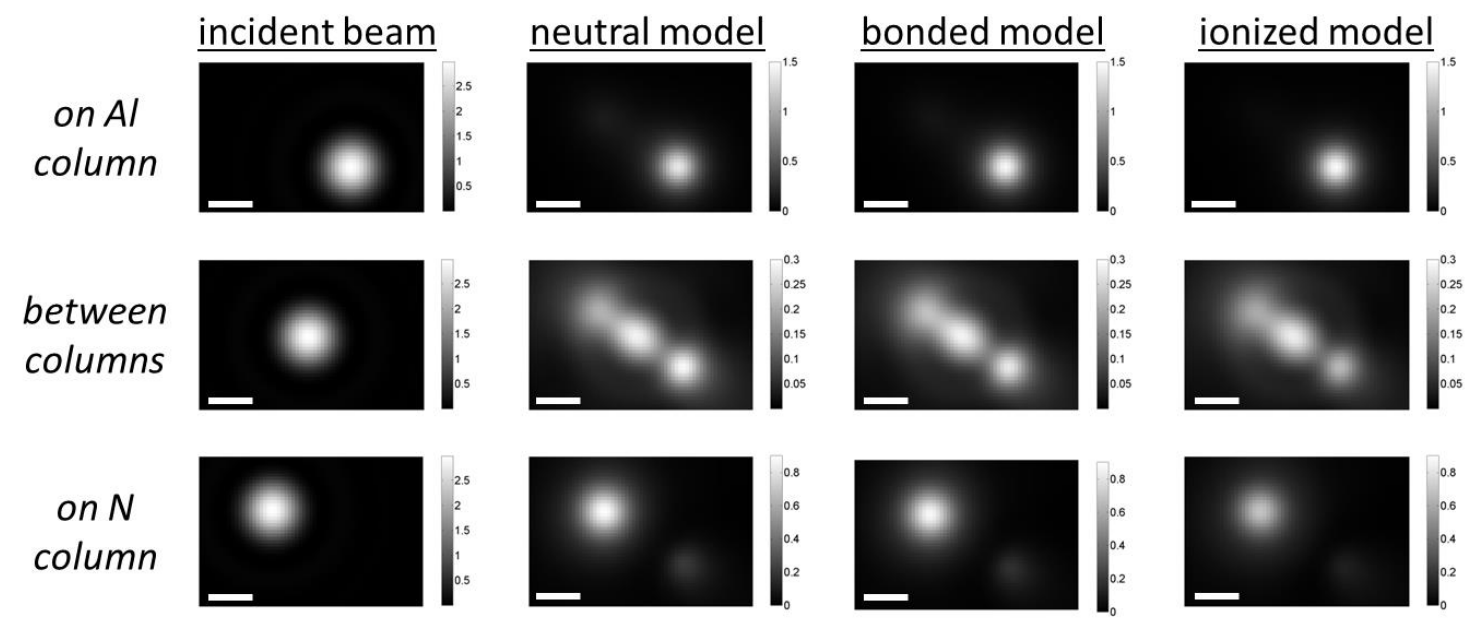

Figure 2. Intensity of a $200 \mathrm{kV}$ probe with convergence semi-angle $25 \mathrm{mrad}$ in <2110>-oriented wurtzite AlN at the incident surface, as well as averaged over $50 \mathrm{~nm}$ depth when modeling the solid as independent neutral atoms, covalently bonded atoms, and independent full-shell ions, respectively (scale bar length $0.5 \AA$, intensity in units of normalized flux $\AA^{2}$ ). The two columns, spaced $1.1 \AA$, apart in projection, interact significantly whether the probe is placed exactly on a column or in between the two. Although the results are subtly sensitive to bonding model, average channeling behavior remains qualitatively the same. 